\title{
Una aproximación a 'según' como estrategia evidencial en El Habla de Monterrey
}

\section{An approach to según as an evidential strategy in El Habla de Monterrey Corpus}

\author{
Liz Maleni Uribe Martínez \\ Universidad Autónoma de Nuevo León, Monterrey, México \\ liz.uribemr@uanl.edu.mx
}

\section{ACCESO ABIERTO / OPEN ACCESS}

Cita: Uribe Martínez, Liz Maleni (2021). Una aproximación a 'según' como estrategia evidencial en $\mathrm{El}$ Habla de Monterrey. Textos en Proceso, 7(1), pp. 1-12. https://doi.org/10.17710/tep.2021.7.

\section{1uribe}

\section{Editoras: Esperanza Alcaide Lara (Universidad de Sevilla) y Ana Pano Alamán (Università di Bologna)}

Recibido: 03/05/2021

Aceptado: 05/07/2021

Conflicto de intereses: La autora ha declarado que no posee conflicto de intereses.

Copyright: () Liz Maleni Uribe Martínez. Esta obra está bajo licencia Creative Commons Reconocimiento 4.0

\section{Resumen}

Se parte de la evidencialidad como categoría gramatical que refiere a la fuente de información en la que un hablante se basa para emitir un enunciado (Aikhenvald, 2003). En el caso del español se habla de estrategias evidenciales (Aikhenvald, 2004). En este trabajo se presenta una primera aproximación al uso de según como estrategia evidencial del español en una muestra de 18 conversaciones semidirigidas pertenecientes al corpus El Habla de Monterrey-PRESEEA ${ }^{1}$. Se analizaron 23 apariciones de según atendiendo a sus efectos sociopragmáticos, es decir, el tipo de evidencialidad que expresa y el grado de compromiso del hablante. Para ello se consideraron los rasgos sociolingüísticos de los hablantes, el contexto en que se dan las conversaciones, así como clasificaciones propuestas por otros autores. Los resultados preliminares sugieren que según puede expresar evidencia directa e indirecta, además se concluye que el hablante puede llegar a reducir el compromiso de lo que dice al combinar según con pronombres personales o con el demostrativo esto. Se propone que estas dos combinaciones permiten asociar la estrategia evidencial con la atenuación, tal como ha sido discutido por otros autores (Albelda, Briz, Cestero, Kotwica, y Villalba, 2014; Briz y Albelda, 2013; Cestero, Albelda, y Briz, 2014).

Palabras clave: evidencialidad, estrategia evidencial, según, atenuación.

\footnotetext{
${ }^{1}$ El corpus El Habla de Monterrey-PRESEEA fue compilado por Lidia Rodríguez Alfano, María Eugenia Flores Treviño y Tzitel Pérez Aguirre. Cuenta con número de Registro SEP 03-2010091313044500-01. ISBN: 978-607-95643-2-2.
} 


\begin{abstract}
Evidentiality is a grammatical category that refers to the source of information on which a speaker relies to make a statement (Aikhenvald, 2003). This work aims to approach the use of según ('according to') as an evidential strategy used in Spanish in a sample of 18 semi-directed conversations of El Habla de Monterrey-PRESEEA corpus. The analysis of 23 uses of según is presented, studying their sociopragmatic functions: the type of evidentiality that según expresses and the notions of the speaker's commitment. Sociolinguistic characteristecs were considered, as well as the context and some categories that have been previously mentioned by other authors. The preliminary results suggest that según can express direct and indirect evidentiality. In addition, the speaker can reduce their commitment by combining según with personal pronouns or with esto ('this'). In this work, it is proposed that those two combinations allow evidentiality to be associated with attenuation, as has been stated by other authors (Albelda, Briz, Cestero, Kotwica \& Villalba, 2014; Briz \& Albelda, 2013; Cestero, Albelda \& Briz, 2014).
\end{abstract}

Keywords: evidentiality, evidential strategy, según, attenuation.

\title{
1. Introducción
}

La evidencialidad corresponde a una categoría gramatical que expresa por medios lingüísticos cuál es la fuente de información en la cual se basa un hablante cuando emite un enunciado (Aikhenvald, 2003, 2004, 2007). En algunas lenguas los marcadores evidenciales se presentan a través de recursos morfológicos, mientras que en otras, la expresión que reporta el significado relacionado con la fuente de información se presenta mediante distintas estrategias evidenciales (Aikhenvald, 2007). Si bien el español no se considera una lengua evidencial debido a la falta de elementos morfemáticos que refieran la fuente de información (Diewald y Smirnova, 2010), se ha concluido que es una lengua que permite el uso de estrategias evidenciales mediante elementos léxicos, tales como: dizque (De la Mora y Maldonado, 2015), al parecer (Kotwica, 2015), el verbo ver (Sanz y Eslava, 2017) y algunas expresiones relacionadas, como por lo visto y se ve (que) (Albelda, 2016, 2018a; Estellés-Arguedas y Albelda, 2017), entre otros. Al respecto, Aikhenvald (2004) propone denominar Estrategias de Evidencialidad a los elementos léxicos o perifrásticos cuya función semántica es establecer la fuente de información o fuente de evidencia.

Este trabajo presenta los primeros hallazgos de un estudio en proceso, el cual pretende analizar el uso de según como estrategia evidencial en el corpus El Habla de Monterrey-PRESEEA, además de mostrar el vínculo que presenta con la atenuación. El corpus completo se compone de 108 conversaciones coloquiales; y para esta primera aproximación, se analizó una muestra de 18 conversaciones, las cuales corresponden a una por cada cruce de las variables sexo (hombre y mujer), edad (tres grupos: de 20 a 34 años, 35 a 54 años y mayores de 55 años) y nivel educativo (bajo, medio y alto). En las siguientes etapas de este trabajo se intentará demostrar que según no solamente es recurrente en el corpus como estrategia evidencial, sino que también guarda una estrecha relación con la disminución de la 
fuerza ilocutiva y con el cuidado de la imagen; es decir, con el concepto de Atenuación (Albelda, 2010; Albelda y Cestero, 2011; Briz, 2012).

\section{Consideraciones teórico-metodológicas}

La evidencialidad difiere en cada lengua, en algunas se distinguen dos fuentes, de primera mano y reportada; mientras que en otros se distinguen hasta seis o más fuentes (cfr. López Martín, 2017). Por lo general la evidencialidad se refiere a acciones que el hablante escucha, huele, siente mediante el tacto o ve, y para considerarse un evidencial, el morfema debe contener una fuente de información que no deja nada a la imaginación (Aikhenvald, 2004).

Se ha reportado que la clasificación que mejor se adapta al español es la de Willett (1998), quien distingue dos grandes categorías: directa e indirecta. Los evidenciales directos indican aquella evidencia en la cual el hablante expresa haber obtenido la información de primera mano. Entre los evidenciales directos se puede distinguir la existencia de evidencia sensorial y evidencia endofórica (Tournade, 1996). La primera indica que la información ha sido obtenida por la vía sensorial, mientras que la segunda señala una fuente de información inaccesible para los sentidos, pero no para el hablante; es decir, es información a la cual solamente el hablante puede acceder mediante su imaginación, estado mental, intenciones o deseos. De manera que vi, escuché u observé son estrategias evidenciales directas sensoriales, ya que son verbos de percepción. Mientras que yo conozco la solución sería un ejemplo de evidencia directa endofórica, pues el hablante está expresando él mismo ser la fuente de información y, al mismo tiempo, está manifestando un estado mental en general, cuya evidencia no puede ser obtenida por medios sensoriales. Una expresión utilizada frecuentemente en el español y que funciona como estrategia evidencial directa endofórica es al parecer (Estellés-Arguedas y Albelda, 2017; Kotwica, 2015).

Por otro lado, los evidenciales indirectos pueden ser inferidos o referidos. Los hablantes utilizan los evidenciales inferidos cuando tienen acceso a la información mediante un proceso de inferencia o deducción, como ocurre en la frase el auto no está en la cochera, no habrán llegado aún ${ }^{2}$, lo cual muestra una deducción del hablante a partir de información con la que ya cuenta. Los evidenciales indirectos referidos ocurren cuando el hablante indica que ha recibido la información a partir de terceros y pueden ser reportativos o citativos. Se ha señalado que los evidenciales reportativos o citativos son frecuentemente formas gramaticalizadas del verbo decir (De Haan, 2001, 2005; Heine y Kuteva, 2002), mientras que según es, por lo general, utilizada como estrategia evidencial indirecta referida cuando es seguida de un pronombre al igual que dizque (De la Mora y Maldonado, 2015).

De acuerdo con su definición formal, según puede funcionar como una preposición o como un adverbio y significa "de acuerdo con, conforme a, de igual o similar manera que [...] tan pronto como, al mismo tiempo que" (El Colegio de México, 2010, p. 1487). Cabe mencionar que Bruyne (1999) clasifica según como una preposición dudosa "debido a que puede aparecer aislada, por ejemplo, en respuestas:-¿Qué harías en esos casos? -Según” (De Bruyne, 1999, p. 697). En

${ }^{2}$ Ejemplos creados por la autora. 
este estudio se consideran todos los usos de según en el corpus seleccionado $\sin$ excluir ninguna de sus funciones.

Según se ha reportado como una estrategia evidencial en distintos estudios (Alonso-Almeida y Adams, 2012; Bermúdez, 2004; Estellés-Arguedas y Albelda, 2014; González-Vergara, 2011; Maldonado y De la Mora, 2015; Nieminen, 2017). En algunas investigaciones se ha optado por analizar la combinación según parece como estrategia evidencial (Estellés-Arguedas y Albelda, 2014; Nieminen, 2017). En primera instancia, en este trabajo se consideran todas las apariciones de según para mostrar su distribución como estrategia evidencial, esto permitirá identificar otras posibles combinaciones en futuras etapas de este estudio.

Alonso-Almeida y Adams (Alonso-Almeida y Adams, 2012) afirman que, en reportes médicos, según hace referencia a investigaciones previas o a evidencias dadas en los resultados de un mismo estudio. Como se observa en el ejemplo 1:

1) Según los resultados de dicho estudio, el SM aumenta 4 veces el riesgo de nuevas complicaciones cardiovasculares o muerte sólo en las mujeres que tienen lesiones coronarias.

En México se ha propuesto que en contextos coloquiales el valor de según se puede extender a reportar rumores, restringir el valor del acto de habla de un interlocutor o incluso emplearlo para pretender hacer algo, como se muestra en los ejemplos 2, 3 y 4 respectivamente (Maldonado y De la Mora, 2015):

2) Según se rumora si no refinamos gasolina aquí/ ¿qué podemos hacer?

3) y le digo/ "bueno/ a ver/ según tú/ ¿cuánto vale tu libertad?”/¿sabes esto cuánto...?

4) y me ponía a leer/ según yo me ponía a leer/ ¡y empezaba!/ a deletrear así/ este/ de una en otra letra

Para poder identificar cuándo una forma lingüística o estructura expresa evidencialidad en el español, Albelda (2018b) ha señalado algunas características que lo indican:

- Señala la fuente de información.

- Opera sobre un alcance proposicional. La indicación de la fuente de información se debe indicar para las proposiciones.

- La evidencia debe ser una justificación para una afirmación de un hecho que está disponible para el hablante.

Es importante señalar que el hecho de que las estrategias evidenciales sean una justificación para una premisa, no implica que se utilicen como una prueba de veracidad. En palabras de Aikhenvald:

Linguistic evidentiality has nothing to do with providing proof in court or in argument, or indicating what is true and what is not, or indicating one's belief. All evidentiality does is supply the information source. The ways in which information is acquired - by seeing, hearing, or in any other way - is its core meaning. (Aikhenvald, 2004) 
Debido a esto, a menudo se confunde la evidencialidad con otros conceptos como la modalidad epistémica o la atenuación. Al respecto Albelda y Estellés (Estellés-Arguedas y Albelda, 2017) afirman lo siguiente:

Esta confusión proviene de que, al no ser obligatoria la expresión de la evidencialidad en español, cuando el hablante decide marcar la fuente de la información, su elección suele responder a estrategias comunicativas concretas, de modo que, junto con el significado básico de 'fuente de información', suelen desarrollarse valores adicionales determinados contextualmente. Entre estos valores se encontrarían la atenuación, la disociación e ironía o la (des)cortesía. (EstellésArguedas y Albelda, 2017).

A partir del análisis de las estrategias evidenciales se puede manifestar un vínculo entre la evidencialidad, la modalidad epistémica, los intensificadores y los atenuadores. Esta investigación busca analizar el uso de según como estrategia evidencial y mostrar la relación que puede presentar con la atenuación. La atenuación es una estrategia discursiva cuyo fin es mitigar el contenido de lo que se expresa (Albelda, 2010; Briz, 2012). Albelda y Cestero (Albelda y Cestero, 2011) definen la atenuación como una función con valor pragmático cuyo objetivo radica en minimizar el efecto de lo dicho. Para identificar la atenuación pragmática, en primer lugar, debe verse involucrada la imagen de uno de los interlocutores o de alguien que no se encuentre presente. La atenuación tiene tres funciones: autoproteger, prevenir y reparar (Albelda et al., 2014; Briz y Albelda, 2013). En este trabajo se consideran todos estos aspectos para el reconocimiento de la atenuación.

\section{Descripción del corpus}

Para la realización de este estudio se analizó una muestra del corpus El Habla de Monterrey-PRESEEA ${ }^{3}$ que en su totalidad consta de 108 conversaciones semidirigidas de hombres y mujeres mayores de 20 años y de tres distintos niveles de instrucción. Se distinguen tres generaciones de hablantes con base en los criterios establecidos por Briz y el Grupo Val.Es.Co (2002, 2003): el grupo 1 se encuentra constituido por hablantes de 20 a 34 años; el grupo 2, por hablantes de 35 a 54 años; y el grupo 3, por hablantes mayores de 55 años. De acuerdo con la propuesta de Briz y VAL.Es.Co (Briz y Grupo Val.Es.Co., 2002) el grado de instrucción también se distribuye en tres grupos: (1) hasta primaria terminada o secundaria trunca (aproximadamente 6 años de instrucción); (2) hasta preparatoria terminada o licenciatura trunca (entre 10 y 12 años de instrucción aproximadamente); y (3) licenciatura terminada o más (aproximadamente 15 años de instrucción o más).

De acuerdo con lo publicado por el PRESEEA (2003), la recolección de datos de sus materiales se hace mediante conversaciones semidirigidas y grabadas con un magnetófono a la vista. Las conversaciones semidirigidas tienen la finalidad de producir una interacción en un tono semiformal a partir de temas diseñados $\mathrm{y}$ planificados, a diferencia de una conversación coloquial, ya que en ellas se utiliza

\footnotetext{
${ }^{3}$ El corpus PRESEEA se recopiló entre 2006 y 2010 y se compone entrevistas guiadas a través de una serie de preguntas que permiten obtener respuestas del entrevistado de distinta tipología textual (expositivas, argumentativas, descriptivas y narrativas).
} 
un tono informal por la ausencia de planificación (Briz, 1998; Molina, 2005). En este trabajo se analiza una muestra de 18 conversaciones que se encuentra disponible en el sitio web oficial del PRESEEA ${ }^{4}$ para descarga libre y gratuita de los audios y transcripciones. Cabe resaltar que esta muestra no es aleatoria, sino que consta de una entrevista por cada cruce de las variables edad, sexo y grado de instrucción como se muestra en la Tabla 1:

Tabla 1. Selección de muestras para estudio

\begin{tabular}{|l|l|l|l|l|l|l|}
\hline Edad & \multicolumn{2}{|l|}{20 a 34 años } & \multicolumn{2}{l|}{35 a 54 años } & \multicolumn{2}{l|}{55 años o más } \\
\hline Sexo & H & M & H & M & H & M \\
\hline Grado de instrucción 1 & 1 & 1 & 1 & 1 & 1 & 1 \\
\hline Grado de instrucción 2 & 1 & 1 & 1 & 1 & 1 & 1 \\
\hline Grado de instrucción 3 & 1 & 1 & 1 & 1 & 1 & 1 \\
\hline
\end{tabular}

\section{Análisis y resultados}

\subsection{Resultados generales}

En un primer acercamiento se encontraron 23 apariciones de según que se clasificaron en estrategia evidencial y no evidencial. Los resultados se pueden observar en la Gráfica 1.

Gráfica 1. Distribución de según en la muestra

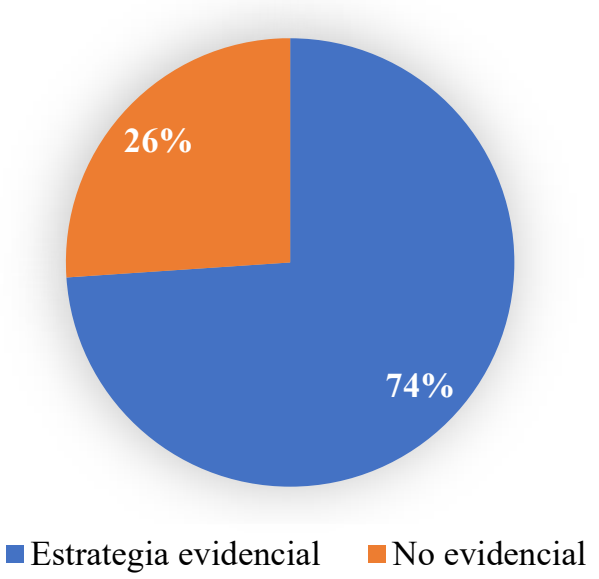

Se observa una mayor tendencia al uso de según como estrategia evidencial. El ejemplo (5) muestra un caso de según como estrategia evidencial, mientras que en el ejemplo (6) se presenta un caso de uso no evidencial:

5) según ahí lo que plantean // los monjes tibetanos le dijeron a la Madre Teresa / (Conversación MONR_H31_076)

6) Después de eso ya se, se pasa, se pasa a sala o al préstamo externo según, según sea. (Conversación MONR_H12_014)

${ }^{4} \underline{\mathrm{http}: / / p r e s e e a .1 i n g u a s . n e t / C o r p u s . a s p x}$ 
Se siguieron los criterios metodológicos propuestos por Albelda (2018b) para identificar la evidencialidad en según (señala la fuente de información, opera sobre un alcance proposicional y ocurre como una justificación para una premisa disponible para el hablante). De manera que en el ejemplo 5, el hablante emplea según para señalar la fuente de información, al contrario del ejemplo 6, donde según no se relaciona con la fuente de información.

\subsection{Valores evidenciales de según}

En el análisis se pudieron identificar ejemplos de los cuatro subtipos de estrategias evidenciales: directa sensorial (7), directa endofórica (8), indirecta referida (9) e indirecta inferida (10):

7) Una vez según yo escuché, escuché así como que respiraban muy cerquita de mí (Conversación MONR_M12_022)

8) La promesa es la que me dieron, la que me dio... lo que según me dio Dios es una promesa para después. (Conversación MONR_H22_052).

9) otros dicen que el señor de la...según esto un señor que los despachó ese día, los muchachos con los que iba él iban discutiendo (Conversación MONR_M22_060)

10) ¿ya no va a venir Miguel Bosé? para irlo a ver I: yo creo que sí viene, yo no creo que sea...según yo era la gira de los treinta años o no sé qué ¿cuántos años tiene de carrera artística? porque ya ves que sacó un $\mathrm{CD}$, el de Papito, que son todas las recopilaciones de sus éxitos y que por eso era la gira... (Conversación MONR_M12_022)

En los ejemplos 7 y 10 se observa la combinación según + yo, la cual es interesante debido a su presencia recurrente en la muestra. Maldonado y De la Mora (Maldonado y De la Mora, 2015) han resaltado el uso de según + yo como un uso de fingimiento del hablante. No obstante, el contexto del ejemplo 7 y 10 sugiere que en ambos casos según funciona como una estrategia evidencial. En el ejemplo 7 el hablante menciona lo que escuchó, por lo que refiere una fuente de información sensorial. Se podría considerar el relacionar esta combinación con un tipo de modalidad epistémica especulativa o deductiva, así como con un valor restrictivo como el descrito por Maldonado y De la Mora (Maldonado y De la Mora, 2015). No obstante, no se ha considerado el incluir este alcance en esta primera etapa de la investigación.

El ejemplo 8 muestra cómo el hablante expresa evidencia directa. La forma en la que se recibió la información (que en este caso es una promesa de Dios) solamente pudo haber sido captada por el hablante debido a la inaccesibilidad a la promesa por medio de los sentidos. Es decir, la promesa a la que el hablante hace referencia pudo haber sido obtenida mediante un estado mental en particular. Es por ello que se le considera un ejemplo de estrategia evidencial directa endofórica.

En el ejemplo 9 se observa otra de las combinaciones analizadas: según + esto. De acuerdo con el uso que se le da en la región, esta expresión hace referencia a un tercero que se omite, de manera que esto no se utilizaría como un pronombre demostrativo, sino que se acercaría más a la función de un pronombre personal, ya que sustituye el nombre de una persona, que en este caso sería la fuente de información. En casos como el ejemplo 9 la combinación según + esto permite ocultar la identidad de la fuente (si es que se conoce) o bien, prescindir de ella (si 
es que se ignora). En cualquiera de los dos casos, el ejemplo cumple con lo necesario para pertenecer a la categoría de estrategia evidencial indirecta referida, ya que se indica que (conocida o ignorada) la fuente de información la brindó un tercero.

Los ejemplos 8 y 9 podrían relacionarse con la propuesta de Maldonado y De la Mora (Maldonado y De la Mora, 2015) del uso de según como un creador de espacio mental (Fauconnier, 1997, 1998; Fauconnier y Turner, 2002). Se trata de una construcción mental permanente que se estructura con elementos, roles, estrategias y relaciones (Fauconnier, 1998). Estos espacios mentales no son parte del lenguaje mismo, sino que corresponden a estrategias que se configuran a partir de elementos gramaticales y pragmáticos. Los espacios mentales son estructuras conceptuales, las cuales se construyen mientras hablamos y representan distintas realidades posibles que se crean bajo un estímulo discursivo (Fauconnier y Turner, 2002). De acuerdo con Pascual, una característica peculiar de los espacios mentales es la siguiente:

Representan realidades posibles en vez de ser reflejos exactos y objetivos de una realidad concreta. Son además configuraciones cognitivas selectivas de dominios de existencia. Es decir, sólo contienen una representación parcial de las entidades y relaciones existentes en un escenario concreto, tal y como éste es percibido, imaginado, recordado, o simplemente entendido por un ente cognoscitivo. (Pascual, 2012, p. 150)

Finalmente, el ejemplo 10 muestra cómo, a partir de ciertos datos conocidos, el hablante expresa un proceso de inferencia a través del cual concluye la información que brinda al inicio del enunciado (la gira musical es debido a los 30 años de carrera artística). Por lo tanto, se trata de una estrategia evidencial indirecta inferida.

La Gráfica 2 a) y b) muestra la distribución de los tipos de estrategia evidencial de según que se encontraron en la muestra. Se puede destacar la alta frecuencia de las estrategias evidenciales indirectas, cuyo uso corresponde al $64.7 \%$.

Gráfica 2. Distribución de los tipos de estrategias evidenciales de según

a)

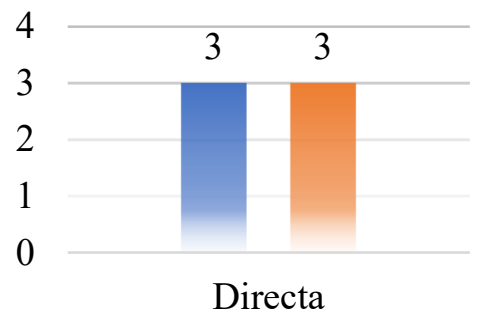

$=$ Sensorial $=$ Endofórica b)

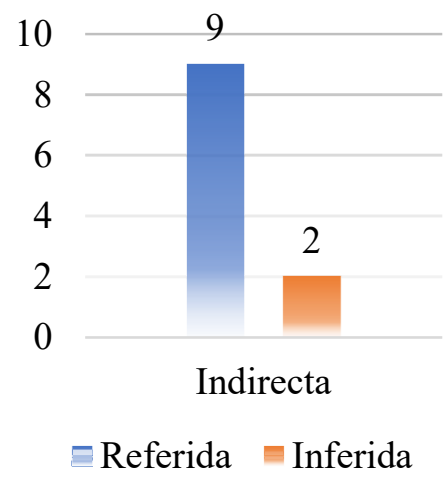


A partir de los datos obtenidos, y como parte de un primer acercamiento a esta investigación, también se consideró relevante el identificar ciertas combinaciones de estructuras que se relacionaran con la aparición de según como estrategia evidencial. El análisis sugiere que la combinación con mayor tendencia a ser utilizada como estrategia evidencial es según + un nombre propio o un pronombre personal en tercera persona. La Gráfica 3 muestra las combinaciones de estructuras que se pueden destacar de la muestra:

Gráfica 3. Combinación de estructuras
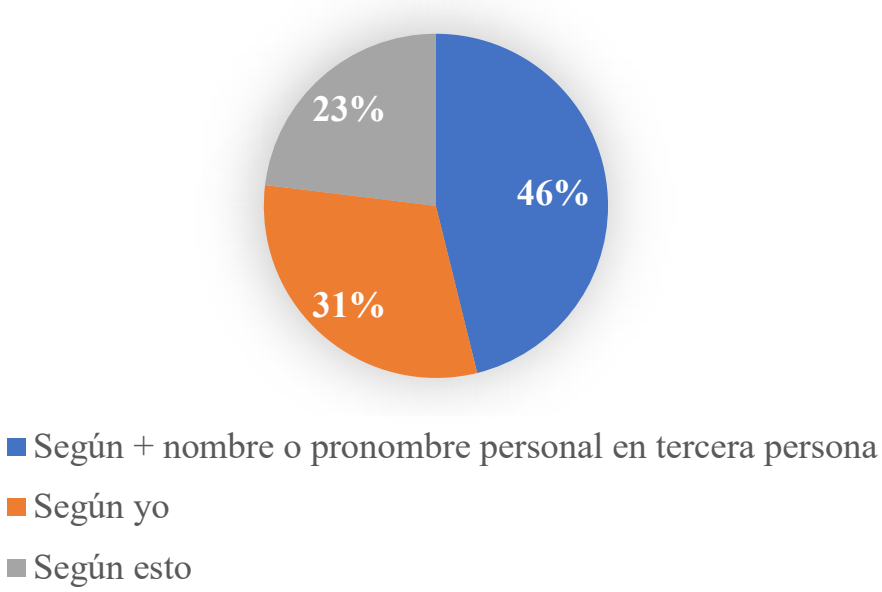

La combinación más empleada en la muestra coincide con los resultados de Nieminem (Nieminen, 2017), autor que relaciona más de la mitad de los casos de según parece con la tercera persona singular. Por otro lado, la combinación según yo ha sido abordada por Maldonado y De la Mora (Maldonado y De la Mora, 2015) como un creador de espacio mental que opera para restringir la validez de lo que se dicesolamente para el hablante, como en los ejemplos 7 o 10. Además, su valor se puede extender a lo pragmático como atenuante, ya que la aserción se disminuye. Finalmente, la combinación según estos e relaciona con el reporte de un rumor o de información que debe ser cuestionada (Maldonado y De la Mora, 2015). En etapas posteriores se trabajará en analizar en profundidad estas tres combinaciones de estructuras, ya que este primer acercamiento sugiere que el valor evidencial de según se puede extender de acuerdo con la estructura en la que se emplea.

\subsection{Según: estrategia evidencial y atenuante}

Si bien el alcance de esta primera etapa de la investigación no incluye un análisis comparativo entre el uso de según como estrategia evidencial y la atenuación, no se puede ignorar el hecho de que en algunos casos se logra observar una disminución del compromiso del hablante con lo dicho, como en los ejemplos 11, 12 y 13:

11) ese fue mi regalo de reyes / llegar a Monterrey / a un proyecto que duraba / según esto tres meses (MONR_H33_099)

12) / nada más seguro de vida / seguro social y fondo de ahorro / según ellos / y nada más (MONR_M21_044) 
13) I: y cada veladora costaba cien pesos / según (MONR_M21_044)

La característica que se busca resaltar en este punto es que en los tres ejemplos se identifica una certeza epistémica baja, por lo que se podría relacionar con un valor pragmático atenuador. En los tres casos se presenta una forma distinta de empleo de según, ya que aparece en combinaciones y posiciones diferentes. La evidencialidad en español ya se ha relacionado con expresiones epistémicas que buscan atenuar (Alonso-Almeida y Adams, 2012; Cornillie, 2007; EstellésArguedas y Albelda, 2017; Kotwica, 2015). Particularmente, son escasos los estudios que relacionan según como evidencial con un significado modal atenuante (Estellés-Arguedas y Albelda, 2014; Maldonado y De la Mora, 2015; Nieminen, 2017). Debido a esto, en etapas posteriores de esta investigación se buscará profundizar sobre las diferentes combinaciones y posiciones en las que según puede funcionar como estrategia evidencial y además, extender su valor a uno atenuante.

\section{Conclusiones}

A partir de este primer acercamiento al análisis de según como estrategia evidencial en El Habla de Monterrey-PRESEEA se destacan tres conclusiones generales que servirán de base para continuar con este estudio. La primera de ellas es que según es capaz de expresar tanto evidencia directa como indirecta. De acuerdo con el análisis, en la muestra seleccionada para este trabajo, en más de la mitad de las ocasiones en las que se utiliza, según tiene un uso como estrategia evidencial indirecta referida. La segunda conclusión es que las combinaciones más frecuentes y que tienden a ser utilizadas como estrategias evidenciales son según + nombre propio o pronombre personal en tercera persona, según yo y según esto. Es por ello que al estudiar una muestra más amplia del corpus se podrá profundizar sobre los usos de estas expresiones, además de relacionarlos con otros conceptos como el de espacios mentales (Fauconnier, 1997, 1998; Fauconnier y Turner, 2002) y la atenuación (Albelda et al., 2014; Briz y Albelda, 2013; Cestero et al., 2014).

La tercera de las conclusiones se relaciona con una de las siguientes etapas de esta investigación, que es el ahondar en la distribución de la atenuación respecto a los tipos de estrategias evidenciales en el corpus. Para algunos autores, la atenuación se relaciona con las estrategias evidenciales indirectas (Estrada, 2008; Sanz y Eslava, 2017), por lo que los datos de este estudio y el análisis del contexto sugieren que es probable que en estudios posteriores de esta investigación se logre identificar que las combinaciones de estructuras de según se asocien con una disminución del compromiso del hablante y con la atenuación.

\section{Referencias}

1. Aikhenvald, A. Y. (2003). Evidentiality in typological perspective. En Aikhenvald, A. Y. y Dixon, R. M. W. (Eds.), Studies in Evidentiality (pp. 1-31). DOI: https://doi.org/10.1075/tsl.54.04aik

2. Aikhenvald, A. Y. (2004). Evidentiality. Oxford: Oxford University Press.

3. Aikhenvald, A. Y. (2007). Information source and evidentiality: what can we conclude?. Rivista Di Linguistica, 19(1), pp. 207-227.

4. Albelda, M. (2010). ¿Cómo se reconoce la atenuación? Una aproximación metodológica basada en el español peninsular hablado. En Orletti, F. y Mariottini, L. (Eds.), (Des)Cortesía en español. Espacios teóricos y metodológicos para su estudio (pp. 1-19). Roma: Università Roma Tre. Disponible en: www.edice.org 
5. Albelda, M. (2016). La expresión de la evidencialidad en la construcción se ve (que). Spanish in Context, 13(2), pp. 237-262. DOI: https://doi.org/10.1075/sic.13.2.04mar

6. Albelda, M. (2018a). ¿Atenuación del compromiso del hablante?: el caso de los evidenciales "por lo visto" y "se ve que.". Rilce. Revista de Filología Hispánica, 34(3), pp. 1179-1214. DOI: https://doi.org/10.15581/008.34.3.1179-214

7. Albelda, M. (2018b). Evidentials as a mark of genre: A study of four oral and written genres. Pragmatics y Society, 9(3), pp. 429-453. DOI: https://doi.org/10.1075/ps.16035.alb

8. Albelda, M., Briz, A., Cestero, A. M., Kotwica, D. y Villalba, C. (2014). Ficha metodológica para el análisis pragmático de la atenuación en corpus discursivos del Español (Es.Por.Atenuación). Oralia, 17, pp. 7-63.

9. Albelda, M. y Cestero, A. M. (2011). De nuevo, sobre los procedimientos de atenuación lingüística. Español Actual: Revista de Español Vivo, 96, pp. 9-40.

10. Alonso-Almeida, F. y Adams, H. (2012). Sentential evidentials in English medical research papers. Revista de Lingüística y Lenguas Aplicadas, 7, pp. 9-21.

11. Bermúdez, F. (2004). La categoría evidencial del castellano: metonimia y elevación de sujeto. Boletín de Lingüística, 22, pp. 3-31.

12. De Bruyne, J. (1999). Las preposiciones. En Bosque, I. y Demonte, V. (dirs.), Gramática descriptiva de la lengua española (Vol. 1, pp. 657-704). Madrid: Espasa.

13. Briz, A. (1998). El español coloquial en la conversación. Barcelona: Ariel.

14. Briz, A. (2012). La (no)atenuación y la (des)cortesía, lo lingüístico y lo social: ¿son pareja? En Escamilla Morales, J. y Vega, G. H. (Eds.), Miradas multidisciplinares a los fenómenos de cortesía y descortesía en el mundo hispánico (pp. 33-75). Barranquilla/Estocolmo: Programa EDICE.

15. Briz, A. y Albelda, M. (2013). Una propuesta teórica y metodológica para el análisis de la atenuación lingüística en español y portugués. La base de un proyecto en común (ES.POR. ATENUACIÓN). Onomázein, 28(2), pp. 288-319. DOI: https://doi.org/10.7764/onomazein.28.21

16. Briz, A. y Grupo Val.Es.Co. (2002). Corpus de conversaciones coloquiales (Oralia). Madrid.

17. Briz, A. y Grupo Val.Es.Co. (2003). Un sistema de unidades para el estudio del lenguaje coloquial. Oralia, 6, pp. 7-61.

18. Cestero, A. M., Albelda, M. y Briz, A. (2014). Estudio coordinado de la "atenuación" en el marco del PRESEEA: propuesta metodológica. XVII Congreso Internacional Asociación de Lingüística y Filología de América Latina (ALFAL 2014) (pp. 26142626). Disponible en: http://www.mundoalfal.org/CDAnaisXVII/trabalhos/R00912.pdf

19. Cornillie, B. (2007). Evidentiality and epistemic modality in Spanish (semilauxiliaries: a cognitive-functional approach. Berlin New York: Gruyter Mouton.

20. De Haan, F. (2001). The Relation between Modality and Evidentiality. Linguistische Berichte, 9, pp. 201-216.

21. De Haan, F. (2005). Encoding speaker perspective: evidentials. En Frajzyngier, Z. et al. (Eds.), Linguistic Diversity and Language Theories (pp. 379-397). Amsterdam/Philadelphia: John Benjamins.

22. De la Mora, J. y Maldonado, R. (2015). Dizque: Epistemics blurring evidentials in Mexican Spanish. Journal of Pragmatics, 85, 168-180. DOI: https://doi.org/10.1016/j.pragma.2015.03.019

23. Diewald, G. y Smirnova, E. (2010). Introduction. Evidentiality in European languages: the lexical-grammatical distinction. En Diewald, G. y Smirnova, E. (Eds.), Linguistic Realization of Evidentiality in European Languages (pp. 1-14). Berlin/New York: Gruyter Mouton.

24. El Colegio de México (2010). Diccionario del español de México. Ciudad de México: El Colegio de México, Centro de Estudios Lingüísticos y Literarios.

25. Estellés-Arguedas, M. y Albelda, M. (2014). Evidentials, politeness and prosody in 4 Spanish: A corpus analysis. Journal of Politeness Research, 10(1), pp. 29-62.

26. Estellés-Arguedas, M. y Albelda, M. (2017). Evidencialidad, atenuación y descortesía en al parecery por lo visto. Influencias del género discursivo. En Cornillie, B. e 
Izquierdo Alegría, D. (Ed.), Gramática, semántica y pragmática de la evidencialidad (pp. 5-28). Pamplona: Ediciones Universidad de Navarra.

27. Estrada, A. (2008). ¿Reforzador o atenuador? "Evidentemente" como adverbio evidencial en el discurso académico escrito. Sintagma, 20, 37-52.

28. Fauconnier, G. (1997). Mappings in Tought and Language. Cambridge: Cambridge University Press.

29. Fauconnier, G. (1998). Mental Spaces. Edinburgh, UK: Cambridge University Press.

30. Fauconnier, G. y Turner, M. (2002). The way we think Conceptual blending and the mind's hidden complexities. New York: Basic Books.

31. González-Vergara, C. (2011). Estrategias gramaticales de expresión de la evidencialidad en el español de Chile. Alpha (Osorno), 32, pp. 149-165.

32. Heine, B., y Kuteva, T. (2002). World Lexicon of Grammaticalization. Cambridge: Cambridge University Press.

33. Kotwica, D. (2015). Evidential al parecer. Between the physical and the cognitive meaning in Spanish scientific prose of the 18th, 19th and early 20th centuries. Journal of Pragmatics, 85, pp. 155-167. DOI: https://doi.org/10.1016/j.pragma.2015.03.021

34. López Martín, J. M. (2017). Estudio macrosintáctico de las estructuras que introducen la fuente enunciativa: como.../ según.../ para.../ en palabras de.../ en opinión de. Círculo de Lingüística Aplicada a La Comunicación, 71, pp. 99-114.

35. Maldonado, R. y De la Mora, J. (2015). Según: a space builder into Mirativity. EHumanista/IVITRA, 8, pp. 488-499.

36. Molina, I. (2005). Estrategias de atenuación en el barrio de Salamanca de Madrid. XIV Congreso Internacional de La Asociación de Lingüistica y Filología de América Latina (ALFAL) (Vol. 1, sección "Dialectología y Sociolingüística"). Monterrey: Universidad Autónoma de Nuevo León.

37. Nieminen, O. (2017). Las locuciones parentéticas por lo que se ve y según parece como marcadores de evidencialidad: sus contextos sintácticos y funciones pragmáticas. Trabajo de fin de Máster: Universidad de Helsinki. Disponible en: https://helda.helsinki.fi/bitstream/handle/10138/225977/Nieminen_Outi_Pro\%20grad u_2017.pdf? sequence $=2$ \&isAllowed $=y$

38. Pascual, E. (2012). Los espacios mentales de la integración conceptual. En Ibarretxe Antuñano, I. y Valenzuela Manzanares, J. (Coords.), Lingüística Cognitiva (pp. 147166). Barcelona: Anthropos.

39. PRESEEA (2003). Proyecto para el Estudio Sociolingüistico del Español de España y América.

40. Sanz, B. E. y Eslava, C. (2017). El uso del verbo ver como estrategia evidencial y de modulación asertiva. En Albelda, M. y Mihatsch, W. (Eds.), Atenuación e intensificación en diferentes géneros discursivos (pp. 111-130). Madrid/Frankfurtam-Main: Iberoamericana/Vervuert.

41. Tournade, N. (1996). Comparaison des systèmes médiatifs de quatre dialectes tibétains (tibétain central, ladakhi, dzonkha et amdo). En Guentchéva, Z. (Ed.), L'énonciation médiatisée (pp. 195-213). Paris/Leuven: Peeters.

42. Willet, T. (1998). A cross-linguistic survey of the grammaticalization of evidentiality. Studies in Language, 12, pp. 57-91. 